\title{
An Elementary Approach to Naturality, Predictability, and the Fundamental Theorem of Local Martingales
}

\author{
Colm O'Cinneide ${ }^{1}$ and Philip Protter ${ }^{2}$
}

\begin{abstract}
We give nontechnical proofs of the predictability of natural processes and the Fundamental Theorem of Local Martingales. Some arguments are shortened through a novel use of an elementary inequality known as "Chebyshev's Other Inequality."
\end{abstract}

Key words and phrases: martingale, stopping time, accessible stopping time, natural process, predictable process

\section{Introduction}

Stochastic integration has become an important tool in the applied sciences and engineering. Familiar examples of this are the famous Black and Scholes option pricing formula and the continuous-time version of the Kalman filter [12]. As the techniques of stochastic integration become more integrated into science and engineering curricula, it becomes important to develop approaches to the subject matter that encompass the most important ideas for applications with a minimum of mathematical background. Such an approach was set out in [13], where a deep and fine analysis of martingales in general was avoided. Our goal in this note is to further simplify the development

\footnotetext{
${ }^{1}$ School of Industrial Engineering, Purdue University, West Lafayette, IN 47907. Supported in part by NSF grants $9414630-D M I I$ and 9714630-DMII.

${ }^{2}$ ORIE-Rhodes Hall, Cornell University, Ithaca, NY 14853, and Department of Mathematics, Purdue University, West Lafayette, IN 47907. Supported in part by NSF grants 9971720-DMS and 9401109-INT, and NSA grant MDA 904-00-1-0035.
} 
presented in [13]. When P.A. Meyer proved what is now known as the Doob-Meyer decomposition $[7,8]$, he used the notion of a natural process in order to obtain uniqueness of the decomposition. Natural processes were indeed natural, as they arose intuitively from a limiting argument applied to Doob's discrete-time decomposition. DoléansDade showed in 1967 [3] that an increasing integrable càdlàg process is natural if and only if it is predictably measurable. This gave rise to elegant, but much less intuitive, proofs of the Doob-Meyer decomposition using the dual predictable projection (see, e.g., $[2,6])$. In [13], the idea of natural processes was resurrected as part of an attempt to keep proofs technically simple and intuitive. A simple proof that a bounded natural process is predictable ([13], p. 121) circumvented the need for some technical theorems of Meyer that were used in Doléans-Dade's proof [3]. There does not, however, appear to be a simple way to extend this proof from bounded to integrable natural processes.

In this note we give a simple proof that natural processes are predictable. Our approach may be explained most easily in the context of the following theorem of Meyer: Theorem 1 (from VII.T49 of [9]): Let $A$ be a natural process. Then (P1) $\Delta A_{T}=0$ a.s. for any totally inaccessible stopping time $T$, and (P2) $\Delta A_{T}$ is $\mathcal{F}\left(T^{-}\right)$-measurable for any predictable stopping time $T$. (See Section 2 for notation and terminology.) We take the following course. In Theorem 4 below, we show that if an adapted, càdlàg, finite-variation process $A$ satisfies conditions (P1) and (P2) then it is predictable, using an elementary result of Bass [1]. Then we prove Theorem 1, thus completing the proof that "natural implies predictable." Our proof of Theorem 1 is made elementary through the use of "Chebyshev's Other Inequality" to establish (P2). This approach, which is an alternative to Doob's ([4], Theorem 2.IV.7(a3), pp. 486-487) or Doléans-Dade's [3], has two attractive features. First, it does not require $A$ to be increasing. Second, it does not require an approximating sequence of continuous processes for $A$. The second point is what makes both Meyer's and Doob's proofs technical and unsuitable for the development in [13]. The present paper may be seen as a complement to Bass's paper [1], in which the DoobMeyer decomposition is proved without recourse to such approximating sequences of continuous processes. Another feature of the approach presented here is that it leads naturally to a simple proof of the Fundamental Theorem of Local Martingales $[11,13]$. 


\section{Background}

We presuppose the material of [13] up to Section 3 of Chapter 3 . Here we review only a few key definitions and results. We are given a filtered probability space $\left(\Omega, \mathcal{F},\left(\mathcal{F}_{t}, t \geq\right.\right.$ $0), P)$, satisfying the usual hypotheses. An adapted càdlàg process $A$ with $A_{0}=0$ is said to be natural if it is of integrable variation and if for every bounded martingale $M$ we have $E[A, M]_{\infty}=0$, where $[A, M]$ denotes the quadratic covariation of $A$ and $M$. By an FV process we mean a càdlàg process with finite variation on compacts. A process $X$ is said to be predictably measurable (or simply predictable) if the function $X(t, \omega): R^{+} \times \Omega \rightarrow R$ is measurable with respect to the $\sigma$-field on $R^{+} \times \Omega$ generated by the a.s. left-continuous adapted processes. Recall that a càdlàg process $X$ is called a quasimartingale if $E\left(X_{0}\right)<\infty$ and the supremum over all partitions $0=t_{0} \leq t_{1} \leq$ $t_{2} \leq \ldots \leq t_{k}$ of the quantity

$$
E\left(\sum_{i=1}^{k}\left|E\left(X\left(t_{i+1}\right)-X\left(t_{i}\right) \mid \mathcal{F}\left(t_{i}\right)\right)\right|\right)
$$

is finite. Here is Rao's theorem [14] on the decomposition of quasimartingales:

Theorem 2 (Rao). A quasimartingale $X$ has a unique decomposition $X=M+A$, where $M$ is a local martingale and $A$ is a natural process.

If $X$ is also an FV process, the process $A$ of Theorem 2 is called the compensator of $X$. We will use the following lemma on continuity of the compensator of certain simple processes that jump only at totally inaccessible stopping times.

Theorem 3. Let $T$ be a totally inaccessible stopping time, and let $V$ be a bounded $\mathcal{F}(T)$-measurable random variable. Let $U_{t}=V I_{(T \leq t)}$ and let $U=M+A$ be the decomposition of $U$ given by Theorem 2. Then $A$ is continuous.

This is a minor extension of Theorem III.11 of [13]. The proof is a bit technical, and involves "energy inequalities." 


\section{$3 \quad$ Predictability of FV Processes}

Theorem 4 below is an elementary condition for predictability of $\mathrm{FV}$ processes, which echoes the theorem of Meyer quoted in the introduction. It is a consequence of Proposition 7.7 of Métivier [6], for example. In the proof presented here, we will use a simple result on predictable stopping times.

Proposition 1. Let $S$ and $T$ be predictable stopping times, and let $R$ be defined by

$$
R=\left\{\begin{array}{lll}
S & \text { if } \quad S \neq T \\
\infty & \text { if } \quad S=T
\end{array}\right.
$$

Then $R$ is again a predictable stopping time.

Bass [1] gives an elementary proof of this, and points out the following useful consequence. Recall first that the graph of a stopping time is the set $\{(\omega, T(\omega)): \omega \in$ $\Omega, T(\omega)<\infty\}$.

Proposition 2. Let $\left(T_{n}\right)$ be a sequence of predictable stopping times. Then there exist another sequence of predictable stopping times $\left(S_{n}\right)$ with disjoint graphs, such that the union of the graphs of the $T_{n}$ 's is the same as the union of the graphs of the $S_{n}$ 's.

Let us write $\Delta X(t)=X(t)-X\left(t^{-}\right)$for the jump in a càdlàg process $X$ at time $t$. Then we have

Theorem 4. Let $A$ be a FV process. If $A$ satisfies conditions (P1) and (P2) of Theorem 1 , then $A$ is predictable.

Proof: Let $A$ be a FV process satisfying (P1) and (P2). In the proof, we carefully exploit the elementary fact that the pointwise limit (in $t$ and $\omega$, except for an evanescent set) of a sequence of predictable processes is predictable. We will not comment further on evanescent sets in the proof. We write $A^{k}$ for the process formed from the jumps of $A$ of magnitude greater than $1 / k$ :

$$
A_{t}^{k}=\sum_{0<s \leq t} \Delta A_{s} I_{\left(\left|\Delta A_{s}\right|>1 / k\right)}
$$

Thus $A-A^{k}$ has no jumps of magnitude greater than $1 / k$. Since $A$ is FV, $A-A^{k}$ converges as $k \rightarrow \infty$ (for all $t, \omega)$ to an adapted continuous process, which is predictable 
by definition. (That $A^{k}$ is adapted is elementary; see [9], IV.44.) Thus to prove that $A$ is predictable, it is enough to prove that $A^{k}$ is predictable. Let $\left(T_{n}\right)$ be the sequence of jump times of $A^{k}$ in increasing order. Since the jump of $A$ at time $T_{n}$ is nonzero on the the entire set $\left(T_{n}<\infty\right),(\mathrm{P} 1)$ implies that $T_{n}$ is accessible. Let $\left(T_{n k}\right)$ be an enveloping sequence of predictable stopping times for $T_{n}$. By Proposition 2 there is a sequence of predictable stopping times $\left(S_{n}\right)$ with disjoint graphs such that the union of the graphs of the $T_{n k}$ 's (over $n$ and $k$ ) is the same as the union of the graphs of the $S_{n}$ 's. As $A^{k}$ is then the sum of the processes $\Delta A^{k}\left(S_{n}\right) I_{\left(S_{n} \leq t\right)}, t \geq 0$, to show that it is predictable it is enough by (P2) to show that the process $V I_{(S \leq t)}, t \geq 0$, is predictable for $S$ a predictable stopping time and $V \in \mathcal{F}\left(S^{-}\right)$. Since such a process is a limit of a sequence of processes of the same form with $V$ bounded, we can assume that $V$ is bounded. Let $\left(R_{n}\right)$ be a sequence of stopping times announcing $S$. Let $V_{n}=E\left(V \mid \mathcal{F}\left(R_{n}\right)\right)$. Then $V_{n} \rightarrow V$ by martingale convergence and the fact that $V \in \mathcal{F}\left(S^{-}\right)=\vee \mathcal{F}\left(R_{n}\right)$. Thus $V I_{(S \leq t)}, t \geq 0$, is the limit of the left-continuous adapted processes $V_{n} I_{\left(R_{n}<t\right)}, t \geq 0$. It follows that $V I_{(S \leq t)}, t \geq 0$, is predictable. This completes the proof.

\section{The Main Result}

The main contribution of this note is a simple proof of

Theorem 5. If a process $A$ is natural, then it is predictable.

Theorem 5 follows at once from Theorems 1 and 4, and so it remains only to prove Theorem 1. The key innovation of this paper is to use a conditional form of Chebyshev's Other Inequality [5] to get condition (P2). More precisely, it is the condition for equality that we need. One version of this inequality is

Lemma 1 (Chebyshev's Other Inequality). Let $X$ be an integrable random variable, and let $F$ be a bounded nondecreasing function. Then

$$
E(X F(X)) \geq E(X) E(F(X))
$$

Moreover, equality holds if and only if $F(X)$ is a.s. constant.

Proof (following [5]): Let $X, Y$ be i.i.d. with the same distribution as $X$. Because $F$ 
is nondecreasing, $(X-Y)(F(X)-F(Y)) \geq 0$. Take expectations on each side of the inequality and multiply out to get (1). Equality in (1) forces the product to be zero a.s. which forces $F(X)=F(Y)$ a.s. since $F$ is nondecreasing, which in turn implies that $F(X)$ is a.s. constant.

Inequality (1) says that $X$ and $F(X)$ are nonnegatively correlated for $F$ increasing. We need the following extension of Lemma 1 for conditional expectations.

Lemma 2. Let $X$ be an integrable random variable, and let $F$ be a bounded nondecreasing function. Let $\mathcal{G}$ be a sub- $\sigma$-field of $\mathcal{F}$. Then

$$
E(X F(X) \mid \mathcal{G}) \geq E(X \mid \mathcal{G}) E(F(X) \mid \mathcal{G}) \text { a.s. }
$$

Moreover, equality holds if and only if $F(X)$ is $\mathcal{G}$-measurable (up to a null set).

Proof: Suppose w.l.o.g. that there exists a random variable $Y$ conditionally independent of $X$ given $\mathcal{G}$, whose conditional distribution given $\mathcal{G}$ is the same as the conditional distribution of $X$ given $\mathcal{G}$. (Such a $Y$ may be constructed on an expanded probability space using regular conditional probabilities, but we omit the routine details.) Let us write $U$ for $F(X)$ and $V$ for $F(Y)$. Then because $F$ is nondecreasing we have $(X-Y)(U-V) \geq 0$ as in the proof of Lemma 1, and so

$$
\begin{gathered}
0 \leq E((X-Y)(U-V) \mid \mathcal{G})=E(X U+Y V-X V-Y U \mid \mathcal{G}) \\
=2(E(X U \mid \mathcal{G})-E(X \mid \mathcal{G}) E(U \mid \mathcal{G})) \quad \text { a.s. }
\end{gathered}
$$

The last equality is by conditional independence and equivalence of the conditional distributions of $X$ and $Y$ given $\mathcal{G}$. This proves (2). If we have equality in (2), we deduce as in the proof of Lemma 1 that $U=V$ a.s. From this it follows that $F(X) \equiv$ $U=E(U \mid \mathcal{G})$, which is $\mathcal{G}$ measurable as required. To see this, note that

$$
E\left((U-E(U \mid \mathcal{G}))^{2}\right)=E((U-E(U \mid \mathcal{G}))(V-E(V \mid \mathcal{G})))=0
$$

by conditional independence.

One final lemma concerning martingales and stopping times will be needed in the proof of Theorem 5. It is well known (see [10], for example), but we provide a proof for the reader's convenience. 
Lemma 3. Let $T$ be a stopping time, and let $Y$ be a bounded $\mathcal{F}(T)$-measurable random variable. Let $Z$ denote $Y-E\left(Y \mid \mathcal{F}\left(T^{-}\right)\right)$, and let $M_{t}=Z I_{(T \leq t)}$. Then $M$ is a càdlàg bounded martingale.

Proof: That $M$ is càdlàg and bounded is obvious. To see that it is a martingale, let $B \in \mathcal{F}_{s}$, and suppose that $t>s$. We must prove that $E\left(M_{t} I_{B}\right)=E\left(M_{s} I_{B}\right)$. We have

$E\left(M_{t} I_{B}\right)=E\left(Z I_{(T \leq t)} I_{B}\right)=E\left(Z I_{(T \leq s)} I_{B}\right)+E\left(Z I_{(s<T \leq t)} I_{B}\right)=E\left(Z I_{(T \leq s)} I_{B}\right)=E\left(M_{s} I_{B}\right)$.

The next-to-last equality is because $E\left(Z \mid \mathcal{F}\left(T^{-}\right)\right)=0$ a.s., and the event

$$
(s<T \leq t) \cap B=(T>s) \cap B-(T>t) \cap B
$$

is in $\mathcal{F}\left(T^{-}\right)$.

Proof of Theorem 5. As noted above, Theorem 5 follows at once from Theorems 1 and 4 , so it remains only to prove Theorem 1 . Towards this end, let $A$ be a natural process. We must prove conditions (P1) and (P2). Proof of Condition (P1): Let T be a totally inaccessible stopping time. Let $F$ be a bounded nondecreasing function on the reals, and let $V=F\left(\Delta A_{T}\right)$. Let $U$ and $M$ be as defined in Theorem 3 for this $T$ and $V$, but denote the compensator of $U$ by $B$ here to distinguish it from the given natural process $A$. Thus $U=M+B$. By Theorem 3 the martingale $M$ is continuous except for a jump of $V$ at $T$. Let $M^{n}$ denote the martingale $M$ stopped when its magnitude first reaches or exceeds $n$. Then, like $M, M^{n}$ is a martingale and is continuous except for a jump at time $T$ of magnitude $V_{n}=V I_{n}$, where $I_{n}$ is the indicator function of the event that the magnitude of $M$ has not reached $n$ before time $T$. Naturality of $A$ then gives $E\left[A, M^{n}\right]=0$ since $M^{n}$ is bounded. But by elementary calculation (see [13], Section II.3)

$$
\left[A, M^{n}\right]=\Delta A_{T} \Delta M_{T}^{n}=\left(\Delta A_{T}\right) V_{n}
$$

Now we take the limit as $n \rightarrow \infty$, observing that $V_{n} \rightarrow V$ a.s., to conclude by dominated convergence that $E\left[\left(\Delta A_{T}\right) V\right]=E\left[\Delta A_{T} F\left(\Delta A_{T}\right)\right]=0$. The choice $F(x)=\operatorname{sign}(x)$ implies that $E\left(\left|\Delta A_{T}\right|\right)=0$, and it follows that $\Delta A_{T}=0$ a.s. Proof of Condition (P2): Let $T$ be a predictable stopping time. Let $F$ be a bounded, strictly increasing function, and let $X$ denote $\Delta A_{T}$. Let $Y=F(X)$, and let $Z$ and $M$ be as in Lemma 3 with this choice of $T$ and $Y$. As $M$ is a bounded martingale and $A$ is natural, we have 
$E[A, M]_{\infty}=0$. Since $M$ has only one jump, at time $T$, it follows that $[A, M]_{\infty}=X Z$, and so

$$
E\left(X\left[F(X)-E\left(F(X) \mid \mathcal{F}\left(T^{-}\right)\right)\right]\right)=0 .
$$

Consider inequality (2) with $\mathcal{F}\left(T^{-}\right)$playing the role of $\mathcal{G}$. Equation (3) implies that the unconditional expectations of each side of (2) agree, and so we must have equality a.s. in (2). Lemma 2 now tells us that $F(X)$ is $\mathcal{G}=\mathcal{F}\left(T^{-}\right)$-measurable. Therefore

$X=\Delta A_{T}$ is also $\mathcal{F}\left(T^{-}\right)$-measurable, since $F$ was chosen to be strictly increasing and hence one-to-one. This establishes (P2). $\mathcal{F}\left(T^{-}\right)$-measurable, It is pleasing that conditions (P1) and (P2) are established directly from the definition of naturality, by constructing a martingale $M$ which allows us to extract information from the condition $E[A, M]=0$. Theorem 5 is one half of the following theorem, which states the equivalence of the concepts of naturality and predictability for processes of integrable variation. It is an immediate consequence of Theorem 5 and Theorem III.23 of [13].

Theorem 6. Let $A$ be an adapted càdlàg process of integrable variation with $A_{0}=0$. Then $A$ is natural if and only if it is predictable.

\section{The Fundamental Theorem of Local Martingales}

The proof of this basic result below is due to Jia-an Yan. We follow the presentation in [13], which in turn is a fuller version of the proof in [11]. Several technicalities may be bypassed when Theorem 5 above is on hand.

Theorem 7 (The Fundamental Theorem of Local Martingales). Let $M$ be a local martingale. Then $M$ is the sum of a local martingale $N$ having bounded jumps and a local martingale $L$ of finite variation on compacts.

Proof: Suppose w.l.o.g. that $M_{0}=0$. By stopping, we can further suppose w.l.o.g. that $M$ is a uniformly integrable martingale. Let $\beta$ be a given positive constant. We set

$$
C_{t}=\sum_{0<s \leq t}\left|\Delta M_{s}\right| I_{\left(\left|\Delta M_{s}\right|>\beta\right)}
$$


We show that $C$ is locally integrable. Define

$$
R_{n}=\inf \left\{t: C_{t} \geq n \text { or }\left|M_{t}\right| \geq n\right\}
$$

Then $\left|\Delta M\left(R_{n}\right)\right| \leq\left|M\left(R_{n}\right)\right|+\left|M\left(R_{n}-\right)\right| \leq\left|M\left(R_{n}\right)\right|+n$. So $C\left(R_{n}\right) \leq C\left(R_{n}-\right)+$ $\left|\Delta M\left(R_{n}\right)\right| \leq\left|M\left(R_{n}\right)\right|+2 n$, which is in $L^{1}$. Since $R_{n} \uparrow \infty, C$ is locally integrable. By stopping, we can now further assume w.l.o.g. that $C$ is integrable: $E\left(C_{\infty}\right)<\infty$. Now define $B$ by

$$
B_{t}=\sum_{0<s \leq t} \Delta M_{s} I_{\left(\left|\Delta M_{s}\right|>\beta\right)}
$$

In fact, $C_{\infty}$ is the total variation of $B$, and, since it is integrable, $B$ is a quasimartingale. By Theorem 2, $B$ has the decomposition

$$
B=L+A
$$

where $L$ is a local martingale and $A$ is a natural process. Since $\left|B_{t}\right|$ and $\left|A_{t}\right|$ are both bounded by the total variation of $B$, namely $C_{\infty}$ (for example by Theorems III.7 and III.8 of [13]), which is integrable, it follows that the local martingales $L$ and

$$
N=M-L=(M-B)+A
$$

are uniformly integrable. We have identified the required decomposition $M=N+L . L$ has integrable (and therefore finite) variation. It remains only to show that the jumps of $N$ are bounded. Let $T$ be any stopping time. If $\Delta A_{T}=0$ a.s. then $\left|\Delta N_{T}\right| \leq \beta$. This is because of (4) and the fact that the jumps of $M-B$ are bounded by $\beta$ in magnitude. Suppose on the other hand that $P(\Lambda)>0$ where $\Lambda=\left(\Delta A_{T}>0\right)$. Let $R=T_{\Lambda}$, which is to say that $R=T$ on $\Lambda$ and $R=\infty$ elsewhere. $R$ is a stopping time as $\Lambda \in \mathcal{F}(T)$. Since the jump of $A$ at time $R$ is nonzero on the the entire set $(R<\infty),(\mathrm{P} 1)$ implies that $R$ is accessible. Thus $R$ has an enveloping sequence of predictable stopping times, and so it suffices to show that $\Delta N(S)$ is bounded by $2 \beta$ for any predictable stopping time $S$. Let $S$ be predictable, and let $\left(S_{n}\right)$ be a sequence of stopping times announcing $S$. Then we have

$$
E\left(N_{S} \mid \bigvee_{n=1}^{\infty} \mathcal{F}\left(S_{n}\right)\right)=\lim _{n \rightarrow \infty} E\left(N_{S} \mid \mathcal{F}\left(S_{n}\right)\right)=\lim _{n \rightarrow \infty} N\left(S_{n}\right)=N\left(S^{-}\right)
$$


so that $E\left(\Delta N_{S} \mid \mathcal{F}\left(S^{-}\right)\right)=0$. It follows that

$$
\begin{aligned}
\Delta N_{S} & =\Delta N_{S}-E\left(\Delta N_{S} \mid \mathcal{F}\left(S^{-}\right)\right) \\
& =\Delta(M-B+A)_{S}-E\left(\Delta(M-B+A)_{S} \mid \mathcal{F}\left(S^{-}\right)\right) \\
& =\Delta(M-B)_{S}-E\left(\Delta(M-B)_{S} \mid \mathcal{F}\left(S^{-}\right)\right) .
\end{aligned}
$$

The final equality is due the fact that $\Delta A_{S} \in \mathcal{F}\left(S^{-}\right)$by naturality of $A$ and condition (P2). Thus $\Delta N_{S}$ is bounded by $2 \beta$, since $\left|\Delta(M-B)_{S}\right|$ is bounded by $\beta$ by definition of $B$. This implies that all the jumps of $N$ are bounded by $2 \beta$, and completes the proof.

\section{$6 \quad$ Remarks on Accessible Stopping Times}

In [13], p. 122, the reader is referred to Doob [4], pp. 483-487, for a proof of what is here Theorem 5. Unfortunately, there is an error in the last step of that proof. The error is in the claim that, for $T$ an accessible stopping time, the process $t \rightarrow I_{[T, \infty)}(t)$ is predictable. To draw this conclusion, $T$ must itself be a predictable stopping time. The error appears on pp. 430-1 and 487 of [4]. Here is a classic example. Let $T$ be a random variable taking values 1 and 2 with equal probability. Let $X(t)=I_{[T, \infty)}(t), t \geq 0$, and equip the process $X$ with its natural filtration. Then $T$ is the jump time of $X$, and is thus a stopping time. It is accessible, since $T$ is either 1 or 2 , and constants are predictable stopping times. To see that $T$ is not predictable, let $S$ be a stopping time with $S<T$ a.s. Then $P(S<1) \geq 1 / 2$, and so for some $a<1$ we have $P(S<a)>0$. But the event $(S<a)$ is in $\mathcal{F}_{a}$, which is the trivial $\sigma$-field for $a<1$. Therefore $P(S<a)=1$. It follows that there cannot exist an announcing sequence $S_{n}$ for $T$, and so we conclude that $T$ is not predictable. That the process $X$ is not predictable may be see by showing that it is not natural, by Theorem 6 . To this end, let $M$ be the martingale $M(t)=(2 T-3) I_{[1, \infty)}(t)$. Then $[X, M]=-1 / 2 \neq 0$, and so $X$ is not natural. 


\section{References}

[1] Bass, R.F. "The Doob-Meyer decomposition revisited." Technical Report, Department of Mathematics, University of Washington, Seattle, WA 98195. 1993.

[2] Dellacherie, C. Capacités et processus stochastiques. Springer, Berlin, Heidelberg, New York, 1972.

[3] Doléans-Dade, C. "Processus croissants naturels et processus croissants trés bien mesurables." CRAS Paris, 264, pp. 874-876, 1967.

[4] Doob, J.L. Classical potential theory and its probabilistic counterpart. Springer, Berlin, Heidelberg, New York, 1984.

[5] Fink, A.M., and M. Jodeit, Jr. "On Chebyshev's other inequality." In Inequalities in Statistics and Probability, Y.L. Tong, editor, IMS Lecture Notes-Monograph Series No. 5, 1982.

[6] Métivier, M. Semimartingales: A Course in Stochastic Processes. De Gruyter, Berlin, 1982.

[7] Meyer, P.A. "A decomposition theorem for supermartingales." Illinois J. Math. 6, 193-205, 1962.

[8] Meyer, P.A. "Decomposition of supermartingales: The uniqueness theorem." Illinois J. Math. 7, 1-17, 1963.

[9] Meyer, P.A. Probability and potentials. Blaisdell, Waltham, MA, 1966.

[10] Meyer, P.A. Un cours sur les intégrals stochastiques. Séminaire Probab. X. Lecture Notes in Mathematics 511, 245-400. Springer, Berlin, Heidelberg, New York, 1976.

[11] Meyer, P.A. "Le Théorèm fondamental sur les martingales locales." Séminaire Probab. XI. Lecture Notes in Mathematics 581, 463-464. Springer, Berlin, Heidelberg, New York, 1977. 
[12] Oksendal, B. Stochastic Differential Equations. Springer-Verlag, Berlin, Heidelberg, New York. 1992.

[13] Protter, P. An Introduction to Stochastic Integration and Differential Equations: A New Approach. Springer-Verlag, 1990.

[14] Rao, K.M. Quasimartingales. Math. Scand. 24, 79-92, 1969. 